\title{
Spin Polarization in Cu Core-Level Photoemission with Linearly Polarized Soft X Rays
}

\author{
Ch. Roth, F. U. Hillebrecht, W. G. Park, H. B. Rose, and E. Kisker \\ Institut für Angewandte Physik, Heinrich-Heine-Universität Düsseldorf, Universitatstrasse 1, D-40225 Dusseldorf, Germany
}

(Received 16 May 1994)

\begin{abstract}
We report on the first observation of electron spin polarization in photoemission from inner shells of a nonmagnetic solid with linearly polarized radiation. The effect is observed in normal emission from the $2 p$ and $3 p$ core levels of $\mathrm{Cu}(001)$ excited with $p$-polarized soft-x-ray radiation at oblique incidence. We interpret the effect in terms of spin-orbit interaction and interference of continuum partial waves. The results are relevant for the understanding of magnetic dichroism and spin polarization in core-level photoemission from ferromagnets.
\end{abstract}

PACS numbers: 71.70.Ej, 79.60.Bm

Since the prediction [1] and the experimental observation [2] of the Fano effect, it is well known that excitation of unpolarized atoms with circularly polarized vacuum ultraviolet (VUV) light can lead to the emission of photoelectrons with a defined spin orientation in the presence of spin-orbit (SO) interaction. Later it was shown that with free atoms or molecules and physisorbed rare-gas monolayers even excitation with linearly polarized or unpolarized light can result in spin polarization in angular-resolved photoemission from outer shells $[3,4]$. This effect has been ascribed to the influence of SO interaction in the bound state or in the continuum together with phase-shift differences in the continuum wave functions. A related effect [5], as well as further spin-polarization phenomena only occurring in non-normal emission geometry [6] or for crystals with special symmetry properties $[7,8]$, has subsequently been observed in photoemission from the valence bands of nonmagnetic solids. In recent years, with the development of intense soft-x-ray synchrotron radiation sources, experimental and theoretical effort has been directed toward the extension of spin resolution to the measurement of the core-level spectra of ferromagnets, for the purpose of understanding multiplet splitting $[9,10]$ and for obtaining element specific information on magnetic ordering in thin film and adsorbate systems [11]. Implicitly it has been assumed that in core-level photoemission with linearly polarized radiation spin polarization in normal emission will only arise due to the magnetic exchange interaction of core electrons with the polarized valence band. In this Letter, we show for the first time that photoelectrons emitted from core levels of a nonmagnetic metal, in this case the $2 p$ and $3 p$ levels of $\mathrm{Cu}(001)$, can be strongly spin polarized upon excitation with linearly polarized light. The polarization results from the interference of partial waves of the photoelectron continuum in the presence of spin-orbit interaction.

The experiments have been carried out using linearly polarized soft-x-ray radiation from the new undulator beam line BW 3 at the $4.5 \mathrm{GeV}$ storage ring DORIS III at HASYLAB (DESY) in Hamburg [12]. The beam line is equipped with a modified SX-700 monochromator and covers the spectral range from $50-2000 \mathrm{eV}$. The sample was a $\mathrm{Cu}(001)$ single crystal, cleaned by sputtering and annealing in UHV as usual. During data acquisition, the pressure in the experimental chamber was $2 \times 10^{-10}$ mbar. A hemispherical photoelectron spectrometer, equipped with an efficient $\mathrm{Fe}(001)$ very-lowenergy electron diffraction (VLEED) spin polarimeter [10], was employed. Linearly $p$-polarized radiation was incident under an angle $\Theta=17^{\circ}$, with respect to the sample surface [see inset of Fig. 1(a)]. The normally emitted photoelectrons were collected with a full acceptance of $8^{\circ}$. The electron-spin analysis was performed for two transversal orientations of the spin quantization axis: first, normal to the reaction plane as defined by the directions of light incidence and electron emission or in the reaction plane. The measured energy distribution curves (EDCs) were normalized to constant photon flux.

Figure 1 displays $\mathrm{Cu} 3 p$ EDCs obtained with a photon energy of $161.3 \mathrm{eV}$. The $\mathrm{Cu} 3 p$ emission is superimposed on a smooth secondary electron background which slightly increases toward higher binding energies. The spin-integrated spectrum [Fig. 1(a)] shows a dominating peak at $74.9 \mathrm{eV}$ binding energy, accompanied by a second smaller one at $77.1 \mathrm{eV}$, attributed to emission from the SO split $p_{3 / 2}$ and $p_{1 / 2}$ states, respectively. Their splitting is $2.2 \mathrm{eV}$, in agreement with literature values $[13,14]$. Figure 1(b) shows spin-resolved $\mathrm{Cu} 3 p$ EDCs obtained with the spin-quantization axis normal to the reaction plane. The EDCs are labeled "spin †" (spin up), or "spin \" (spin down), respectively, according to the orientation of the $y$ component of the electron spin. Strong spin polarization of the $\mathrm{Cu} 3 p$ emission is evident. The polarization is opposite in the two SO split components and is approximately 2 times stronger in the $p_{1 / 2}$ than in the $p_{3 / 2}$ part of the spectrum. The spin-up EDC exhibits clearly resolved peaks of comparable intensity in the $p_{1 / 2}$ and the $p_{3 / 2}$ parts, while the spin-down channel is dominated by the $p_{3 / 2}$ peak, with the $p_{1 / 2}$ state appearing as a small shoulder on the high-binding energy side. Figure 1(c) shows the difference spectrum of the two spin-resolved EDCs of Fig. 1(b). The intensities in the two spin channels integrated over the whole SO split spectrum are equal within $1 \%$ of the total spin-integrated $3 p$ emission [15]. This 


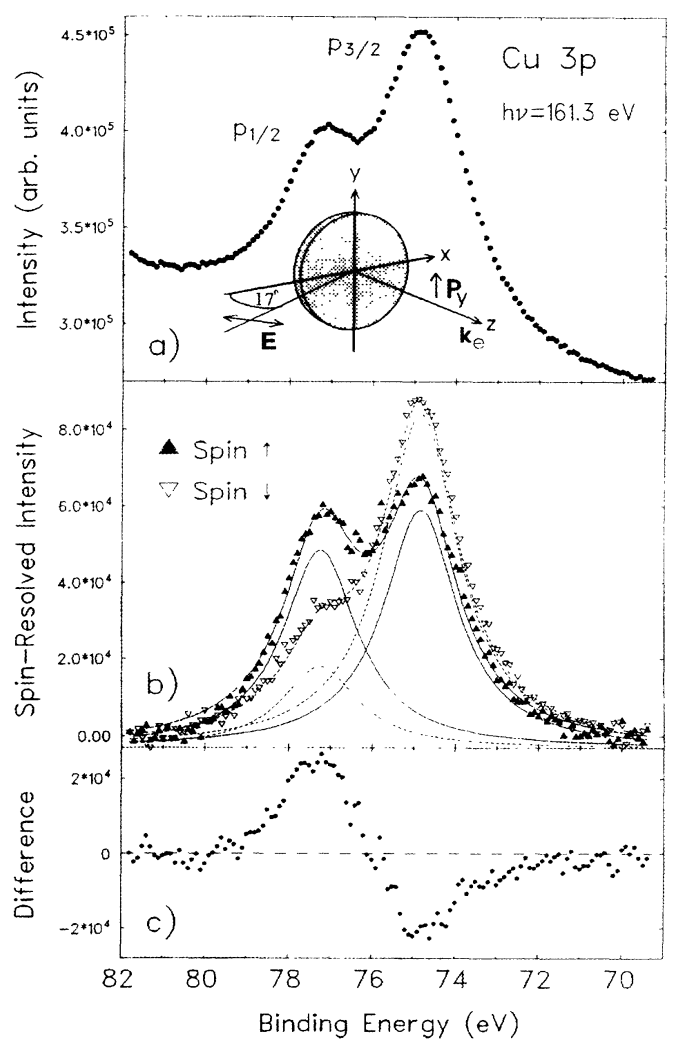

FIG. 1. $\mathrm{Cu} 3 p$ spectra obtained with $h \nu=161.3 \mathrm{eV}$. (a) Spin-integrated energy distribution curve with $\left(p_{1 / 2}, p_{3 / 2}\right)$ spin-orbit (SO) splitting resolved. Inset: Geometry of the experiment. Linearly $p$ polarized radiation is incident on a $\mathrm{Cu}(001)$ crystal under an angle $\Theta=17^{\circ}$, with respect to the sample surface. Normally emitted electrons are collected with $8^{\circ}$ full acceptance. The transversal electron-spin polarization is measured with the quantization axis either normal $\left(\mathbf{P}_{y}\right)$ to the reaction plane, defined by the directions of light incidence and electron emission, or in the reaction plane (along $x$ ). (b) Spin-resolved EDCs obtained with the spin quantization axis normal to the reaction plane, after background subtraction. The spin resolved EDCs are labeled spin $\uparrow$ (spin up) or spin $\downarrow$ (spin down) according to the orientation of the $y$ component of the electron spin, parallel or antiparallel to the $y$ axis of the coordinate system. The lines show the result of a decomposition into 4 Lorentzians (2.2 eV FWHM). (c) Difference spectrum obtained from the EDCs in (b). Vertical scale units are arbitrary but equal in (a), (b), and (c).

evidences the fundamental role of core-hole SO interaction for the mechanism leading to the spin polarization: Without experimentally resolving the SO splitting, no polarization would be observed in the spectrum.

The $x$ component of the electron spin polarization in the $\mathrm{Cu} 3 p$ spectrum, measured at the same photon energy, is virtually zero. This is in agreement with symmetry arguments based on parity conservation [16] forbidding the existence of spin polarization in a mirror plane of the system for an unpolarized target; so that $\mathbf{P}_{x}\left(=\mathbf{P}_{z}\right)=0$. In contrast, the $(y, z)$ plane is a mirror plane only for $\Theta=0^{\circ}$ or $\Theta=90^{\circ}$, and thus spin polarization normal to the reaction plane (i.e., $\mathbf{P}_{y}$ ) can exist for an angle of incidence $0^{\circ}<\Theta<90^{\circ}$. This is actually observed for $\Theta=17^{\circ}$ (Fig. 1). From the symmetry considerations it also follows that the polarization integrated over all emission directions should vanish.

Our experimental results show a striking analogy to theoretical and experimental results on photoionization of free rare-gas atoms. Spin polarization in photoemission from unpolarized isotropic atoms with linearly polarized light was first discussed theoretically by Cherepkov and by Lee [3]. The polarization vector $\mathbf{P}$ is found to be parallel to $[\mathbf{k} \times \mathbf{E}$ ], the vector product of the photoelectron momentum $\mathbf{k}$, and the light polarization vector $\mathbf{E}$ and vanishes if integrated over all emission directions, and also for $\mathbf{k} \| \mathbf{E}$ and $\mathbf{k} \perp \mathbf{E}$. Spin polarization should therefore only be observable in a noncoplanar experimental arrangement of $\mathbf{k}, \mathbf{E}$, and the quantization axis of the spin detector, as is also seen in our experiment, in accord with the general symmetry considerations given above. The existence of a spin polarization parallel to $[\mathbf{k} \times \mathbf{E}]$ in photoemission from the valence shells of rare-gas atoms has been experimentally verified by Schönhense et al. [4]. According to theory, spin polarization can arise due to interference between the final-state continuum wave functions with angular-momentum quantum number $1+1$ and $1-1$, which are allowed by dipole selection rules, in the presence of SO interaction in the bound state with $1>0$. The sign of the polarization is reversed for the SO split components of the spectrum, with the polarization in the ${ }^{2} P_{1 / 2}$ final ionic state 2 times stronger than in the ${ }^{2} P_{3 / 2}$ state.

A similar sign reversal is observed in the $\mathrm{Cu} 3 p$ spectra in our experiment (Fig. 1). The switching of the spinpolarization sign is also seen in photoemission from the $\mathrm{Cu} 2 p$ core level, where the SO split components are well separated in energy (Fig. 2). Photoelectrons emitted from the $2 p$ levels show spin polarization normal to the reaction plane, as in the $3 p$ level. In the $p_{1 / 2}$ component there is a surplus of emission in the spin-up channel, while in the $p_{3 / 2}$ component spin-down emission prevails.

According to theoretical treatment [17] for free atoms, the spin polarization should show a dependence on the photon energy, given by

$$
P \propto \frac{R_{2} R_{0} \sin \left(\delta_{2}-\delta_{0}\right)}{R_{0}^{2}+2 R_{2}^{2}} \frac{1}{1+\beta P_{2}(\cos \Theta)},
$$

where $R_{2}$ and $R_{0}$ are the radial parts (integrals) of the dipole matrix elements for the allowed transitions to the continuum states, $\delta_{2}-\delta_{0}$ is the phase difference of the continuum wave functions, $\beta$ is the well-known asymmetry parameter, and $P_{2}$ a Legendre polynomial. As a first test for the applicability of this atomic model to core-level photoemission from solids, polarization values for the $\mathrm{Cu}$ $3 p$ levels obtained along this approach [18] are shown together with experimental data in the photon energy range $110-210 \mathrm{eV}$ in Fig. 3. Calculated and experimental values agree in the order of magnitude, in the sign of the 


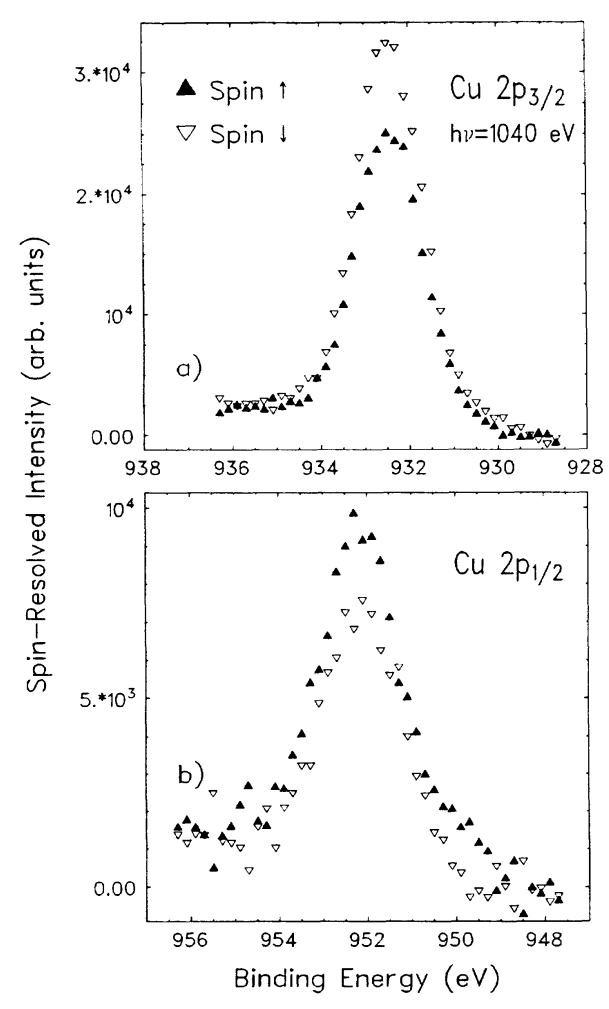

FIG. 2. Spin-resolved $\mathrm{Cu} 2 p$ spectrum obtained with the spinquantization axis normal to the reaction plane $(h \nu=1040 \mathrm{eV})$. The EDCs are labeled as in Fig. 1. (a) $2 p_{3 / 2}$ and (b) $2 p_{1 / 2}$ spectrum.

polarization for the $p_{1 / 2}$ and $p_{3 / 2}$ emission, respectively, and in the observation of a higher polarization for the $p_{1 / 2}$ component. The measured energy dependence roughly agrees with the calculation. However, the maximum of the polarization is shifted to higher photon energy; although this apparent discrepancy may also be due to the coarse energy intervals of the calculation [18]. Quantita-

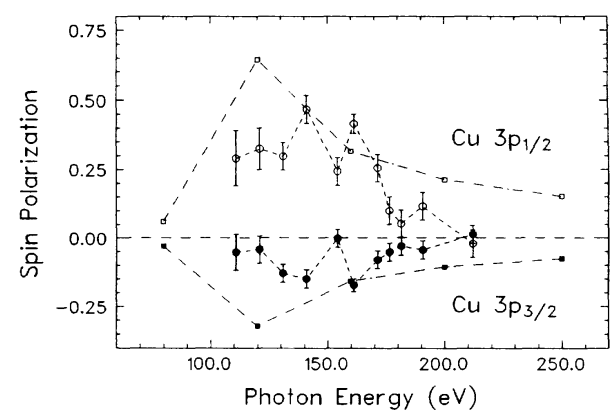

FIG. 3. Photon energy dependence of the $\mathrm{Cu} 3 p$ spin polarization. Circles: Experimental results obtained with the help of a fitting procedure as shown for $h \nu=161.3 \mathrm{eV}$ in Fig. 1. Error bars represent estimated uncertainties due to statistics, instrumental asymmetries, and uncompleted knowledge of the shape of the secondary electron background. Squares: Calculated values; the connecting line interpolates these values linearly. The formulas derived for free atoms in [17], and parameters tabulated by Goldberg et al. [18] were used. tively, strong deviations are evident particularly at photon energies below $160 \mathrm{eV}$. Here the ratio $P\left(p_{3 / 2}\right) / P\left(p_{1 / 2}\right)$ differs considerably from -0.5 , the value obtained within the model. Also, the measured photon energy dependence is stronger than calculated. The deviations might be due to the neglect of many electron correlations and to relativistic effects, i.e., the spin dependence of the transition matrix elements and the SO interaction in the photoelectron continuum. SO interaction in the continuum can lead to phase differences of outgoing partial waves with different total angular momentum. In the vicinity of the Cooper minimum of the $3 p$ photoionization cross section at $h \nu \approx 105 \mathrm{eV}$, their interference can result in additional polarization effects $[19,20]$, leading to deviations of the $p_{3 / 2} / p_{1 / 2}$ polarization ratio from the nonrelativistic value, as observed here.

In the normal emission geometry employed in our experiment, spin polarization due to spin dependent transmission through the crystal surface or photoelectron diffraction [6] can be excluded. Hypothetical polarization effects analogous to those observed in valence-band photoemission from crystals with special symmetry properties $[7,8]$ cannot contribute either, since they are restricted to noncentrosymmetric crystals [7] or crystal surfaces with a threefold symmetry [8]. Our results suggest that the mechanism responsible for spin polarization in the photoionization of free atoms and molecules, based on corehole SO interaction and interference of continuum partial waves [3], also leads to the observed spin polarization in core-level photoemission from a nonmagnetic solid. Consequently, spin polarization has to be regarded as a rather general phenomenon not only in atomic photoionization or valence-band photoemission but also in photoemission from inner shells of solids.

Our results are important for the understanding of spin polarization and magnetic dichroism in core-level photoemission from ferromagnets. There the spin-orbit (SO) induced spin polarization, investigated here for $\mathrm{Cu}(001)$, can be present simultaneously with spin polarization originating from ferromagnetism due to core-level exchange splitting, which is produced by core-hole exchange interaction with the polarized valence bands. These two origins of spin polarization have to be distinguished carefully for an analysis of magnetic properties by spin-polarization measurements. Furthermore, from the combined action of SO induced spin polarization and magnetic exchange splitting a dependence of spin-integrated line shapes on the sign of the magnetization can result, if the magnetization vector has a nonzero component collinear to the axis of the SO induced spin polarization. The existence of this effect [magnetic linear dichroism in the angular distribution of photoelectrons (MLDAD)] has been shown recently in angular resolved $3 p$ [21-23] photoemission from ferromagnetic $\mathrm{Fe}$.

The method presented here offers the possibility to investigate SO interaction in the outer core levels of the early $3 d$ transition metals, where SO interaction is small 
and the spin-integrated line shape does not show SO splitting. An alternative approach has been proposed recently [24]. Excitation of core electrons with SO interaction in magnetic or nonmagnetic solids with circularly polarized radiation should lead to spin-polarized emission, with the sign of the polarization dependent on the total angular momentum of the core hole. As we show in this work, obviously similar information can be obtained in angular resolved photoemission by using linearly polarized radiation, if a suitable experimental geometry is chosen. In this way, the experimental difficulties arising due to the lower intensity and incomplete polarization of circularly polarized soft $x$ rays are avoided. Measurement of the spin polarization should also allow the investigation of the phase shift differences and matrix elements for the dipoleallowed transitions to the $(1-1,1+1)$ photoelectron continuum waves [25]. A further potential application is the utilization of core levels of a nonmagnetic solid as an internal source of spin-polarized electrons, e.g., for experiments on spin-dependent photoelectron diffraction or on the spin dependence of inelastic scattering processes in ferromagnetic overlayers. In all cases, the spin polarization produced by $p$-polarized radiation, as employed in this experiment, must also be observable with unpolarized radiation due to its $p$-polarized component. Therefore in principal even the use of laboratory $x$-ray sources should be possible, if a photon energy appropriate for the specific core level under investigation is available.

We gratefully acknowledge the support of Th. Möller and F. Federmann of HASYLAB in the operation of the BW 3 beam line. This work was supported by the Federal Ministry for Research and Technology (BMFT) under Grant No. 05 5PFDA B 3 and by the DFG (SFB 166).

[1] U. Fano, Phys. Rev. 178, 131 (1969).

[2] U. Heinzmann, J. Kessler, and J. Lorenz, Z. Phys. 240, 42 (1970).

[3] N. A. Cherepkov, Zh. Eksp. Teor. Fiz. 65, 933 (1973) [Sov. Phys. JETP 38, 463 (1974)]; C. M. Lee, Phys. Rev. A 10, 1598 (1974).

[4] U. Heinzmann, G. Schönhense, and J. Kessler, Phys. Rev. Lett. 42, 1603 (1979); G. Schönhense, V. Dzidzonou, S. Kaesdorf, and U. Heinzmann, Phys. Rev. Lett. 52, 811 (1984); G. Schönhense, Phys. Scr. T31, 255 (1990).

[5] E. Tamura and R. Feder, Europhys. Lett. 16, 695 (1991); B. Schmiedeskamp, N. Irmer, R. David, and U. Heinzmann, Appl. Phys. A 53, 418 (1991).

[6] J. Kirschner, R. Feder, and J. F. Wendelken, Phys. Rev. Lett. 47, 614 (1981); H.P. Oepen, K. Hünlich, and J. Kirschner, Phys. Rev. Lett. 56, 496 (1986).
[7] S. F. Alvarado, H. Riechert, and N. E. Christensen, Phys. Rev. Lett. 55, 2716 (1985).

[8] E. Tamura, W. Piepke, and R. Feder, Phys. Rev. Lett. 59, 934 (1987); B. Schmiedeskamp, B. Vogt, and U. Heinzmann, Phys. Rev. Lett. 60, 651 (1988).

[9] C. Carbone and E. Kisker, Solid State Commun. 65, 1107 (1988); C. Carbone, T. Kachel, R. Rochow, and W. Gudat, Solid State Commun. 77, 619 (1991); D. G. van Campen and L. E. Klebanoff, Phys. Rev. B 49, 2040 (1994).

[10] F. U. Hillebrecht, R. Jungblut, and E. Kisker, Phys. Rev. Lett. 65, 2450 (1990); R. Jungblut, Ch. Roth, F. U. Hillebrecht, and E. Kisker, Surf. Sci. 269/270, 615 (1992).

[11] B. Sinkovic, P. D. Johnson, N. B. Brookes, A. Clarke, and N. V. Smith, Phys. Rev. Lett. 65, 1647 (1990); F. U. Hillebrecht, Ch. Roth, R. Jungblut, E. Kisker, and A. Bringer, Europhys. Lett. 19, 711 (1992); G. A. Mulhollan, A. B. Andrews, and J. L. Erskine, Phys. Rev. B 46, 11212 (1992).

[12] C. U. S. Larsson, A. Beutler, F. Federmann, A. Rieck, S. Verbin, and T. Möller (to be published); T. Möller, Synchrotron Radiation News 6, No. 4, 16 (1993).

[13] J.C. Fuggle, J. Electron Spectrosc. Relat. Phenom. 21, 275 (1980).

[14] E. Jensen, R. A. Bartynski, S. L. Hulbert, E. D. Johnson, and R. Garret, Phys. Rev. Lett. 62, 71 (1989).

[15] In order to determine the total $3 p$ intensity, a linear shape of the secondary electron background under the $3 p$ emission was assumed.

[16] J. Kessler, Polarized Electrons (Springer-Verlag, Berlin, 1985); E. Tamura and R. Feder, in [5].

[17] N. A. Cherepkov, Adv. At. Mol. Phys. 19, 395 (1983).

[18] For the calculation, tabulated values for the asymmetry parameter, the radial dipole matrix elements, and the phase shifts obtained within the one-electron central potential model for selected photon energies were taken from S. M. Goldberg, C. S. Fadley, and S. Kono, J. Electron Spectrosc. Relat. Phenom. 21, 285 (1981). A zero of the polarization to be expected near the Fano minimum $(\approx 105 \mathrm{eV})$ is not evident in the calculated values since there were no parameters for the calculation available near this photon energy.

[19] K.-N. Huang, W. R. Johnson, and K. T. Cheng, Phys. Rev. Lett. 43, 1658 (1979).

[20] G. Schönhense et al. (1984) (Ref. [4]).

[21] Ch. Roth, F. U. Hillebrecht, H. B. Rose, and E. Kisker, Phys. Rev. Lett. 70, 3479 (1993).

[22] Ch. Roth, H. B. Rose, F. U. Hillebrecht, and E. Kisker, Solid State Commun. 86, 647 (1993).

[23] F. Sirotti and G. Rossi, Phys. Rev. B 49, 15682 (1994).

[24] B. T. Thole and G. van der Laan, Phys. Rev. Lett. 67, 3306 (1991).

[25] U. Heinzmann, J. Phys. B 13, 4367 (1980). 


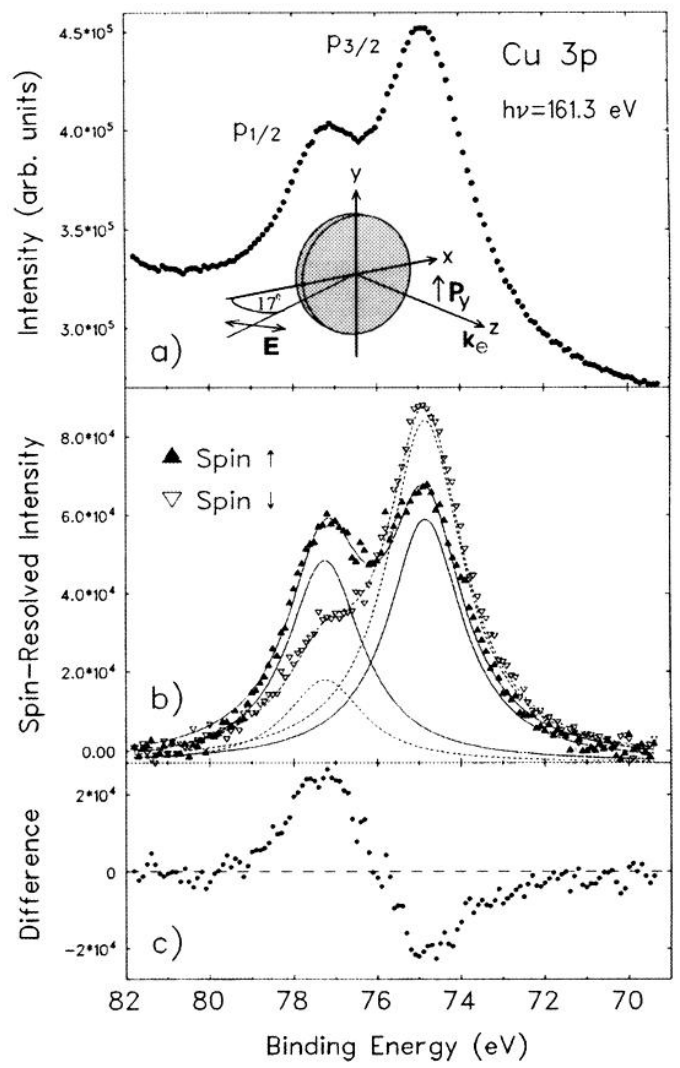

FIG. 1. $\mathrm{Cu} 3 p$ spectra obtained with $h \nu=161.3 \mathrm{eV}$. (a) Spin-integrated energy distribution curve with $\left(p_{1 / 2}, p_{3 / 2}\right)$ spin-orbit (SO) splitting resolved. Inset: Geometry of the experiment. Linearly $p$ polarized radiation is incident on a $\mathrm{Cu}(001)$ crystal under an angle $\Theta=17^{\circ}$, with respect to the sample surface. Normally emitted electrons are collected with $8^{\circ}$ full acceptance. The transversal electron-spin polarization is measured with the quantization axis either normal $\left(\mathbf{P}_{y}\right)$ to the reaction plane, defined by the directions of light incidence and electron emission, or in the reaction plane (along $x$ ). (b) Spin-resolved EDCs obtained with the spin quantization axis normal to the reaction plane, after background subtraction. The spin resolved EDCs are labeled spin $\uparrow$ (spin up) or spin $\downarrow$ (spin down) according to the orientation of the $y$ component of the electron spin, parallel or antiparallel to the $y$ axis of the coordinate system. The lines show the result of a decomposition into 4 Lorentzians (2.2 eV FWHM). (c) Difference spectrum obtained from the EDCs in (b). Vertical scale units are arbitrary but equal in (a), (b), and (c). 